ARTÍ́CULO DE REVISIÓN

\title{
Modelos murinos para el estudio de la diabetes tipo 2: una revisión sistemática
}

\author{
Amelia Martí1 ${ }^{1 *} *$ y Cristina Tapiz ${ }^{1}$
}

${ }^{1}$ Departamento de Nutrición, Ciencia de los alimentos y Fisiología, Universidad de Navarra, Pamplona; ${ }^{2}$ Departamento de Nutrición, Ciencia de los Alimentos y Fisiología, Instituto Navarro de Investigación en Salud (IdiSNA), Pamplona; ${ }^{3}$ Centro de Investigación Biomédica en Red Fisiopatología de la Obesidad y Nutrición (CIBEROBN), Instituto of Salud Carlos III, Madrid. España

\section{RESUMEN}

En los últimos años, ha habido un número creciente de estudios centrados en la diabetes mellitus, incluyendo su fisiopatología, complicaciones y tratamiento. Entre los distintos tipos, la diabetes mellitus tipo 2 (DMT2) es la causa más común. Sus manifestaciones incluyen resistencia a la insulina y posterior disfunción de las células $\beta$ pancreáticas, y su etiología comprende tanto factores genéticos como del estilo de vida. Debido a ello, esta revisión sistemática tiene como objetivo examinar distintos modelos animales de DMT2 para comprender los factores que influyen en su desarrollo y complicaciones, y obtener nueva información sobre su manejo y tratamiento en humanos. En resumen, la utilidad de los modelos animales se basa en que manifiestan diferentes aspectos de la DMT2 humana, pero no existe ninguno que la reproduzca en su totalidad.

Palabras clave: Resistencia a la insulina. Diabetes mellitus tipo 2. Terapia nutricional. Modelos animales de enfermedad. Ratón.

\section{ABSTRACT}

In recent years, there have been an increasing number of studies focusing on diabetes mellitus, its pathophysiology, complications and treatment. Among the different types, type 2 diabetes mellitus (T2DM) is the most common. Its manifestations include insulin resistance and subsequent $\beta$ cell secretory dysfunction, and its etiology comprises both genetic and lifestyle factors. Thus, this systematic review aims to examine different animal models of T2DM in order to understand the factors that may influence its development and complications, and thus gain new information about its management and treatment in humans. In short, the utility of animal models is based on the fact that they manifest different aspects of human T2DM, but none reproduces it completely. (Rev ALAD. 2019;9:165-78) Corresponding author: Amelia Martí, amarti@unav.es

Key words: Insulin resistance. Type 2 diabetes mellitus. Nutrition therapy. Animal disease models. Murine. 


\section{DIABETES MELLITUS, RESISTENCIA A LA INSULINA Y DIABETES MELLITUS TIPO 2}

La diabetes mellitus puede definirse como una enfermedad crónica que afecta a 422 millones de personas en todo el mundo, que es una causa importante de discapacidad a largo plazo y tiene un impacto económico considerable. Por lo tanto, puede considerarse una de las enfermedades más prevalentes, cuya incidencia aumenta con la edad'. Varios procesos patológicos están involucrados en su desarrollo y su etiología se considera poligénica en lugar de monogénica. Se caracteriza por hiperglucemia, debida a defectos en la producción, acción tisular de insulina o ambos ${ }^{2}$.

La insulina, hormona pancreática responsable de regular la homeostasis de la glucosa, lípidos y proteínas, es liberada a circulación sanguínea en respuesta a un aumento de glucosa en sangre ${ }^{3}$. Una vez en sangre, se une a sus receptores con actividad tirosina cinasa situados en diversos tejidos y órganos, como músculo, tejido adiposo, hígado, páncreas, endotelio, gónadas, riñón, huesos y cerebro³. Tras atravesar la barrera hematoencefálica, lleva a cabo una serie de acciones que incluyen el control de la ingesta de alimentos y los efectos sobre las funciones cognitivas ${ }^{4}$.

Su diagnóstico se produce cuando los niveles de glucosa en ayunas superan los $126 \mathrm{mg} / \mathrm{dl}$ o cuando los niveles glucemia superan los $200 \mathrm{mg} / \mathrm{dl}$ tras una determinación al azar o a las 2 horas de una sobrecarga oral de glucosa. Sus síntomas principales incluyen micción frecuente, aumento de la sed, hambre y pérdida de peso. La clasificación de la diabetes incluye diabetes mellitus tipo 1, diabetes mellitus tipo 2 (DMT2) y diabetes mellitus gestacional².
La DMT2 resulta de una producción de insulina alterada por parte de las células $\beta$ pancreáticas o de una liberación de insulina alterada en respuesta a la hiperglucemia. Comprende la mayoría de los casos, ya que supone el $90 \%$ del total de estos, y su prevalencia aumenta con la edad, aunque también se ha observado en individuos jóvenes 5 . La DMT2 está asociada con la resistencia a la insulina, de tal forma que, en condiciones normales, como se ha comentado anteriormente, la insulina mantiene los niveles de glucosa en sangre estables. Sin embargo, cuando se establece la resistencia a la insulina, esta no ejerce su acción tisular adecuadamente y se produce hiperglucemia. Al principio, las células $\beta$ pancreáticas de los islotes de Langerhans aumentan la síntesis y la secreción de insulina para compensar la situación. Pero conforme pasa el tiempo, no pueden mantener el ritmo y son incapaces de producir suficiente insulina para mantener la glucosa sanguínea en niveles normales, lo que conduce al desarrollo de prediabetes o diabetes ${ }^{3}$.

El control inadecuado de los niveles sanguíneos de glucosa conduce a complicaciones crónicas que incluyen microangiopatía (retinopatía, nefropatía, neuropatía), macroangiopatía (enfermedad coronaria, enfermedad cerebrovascular, enfermedad vascular periférica) y, posiblemente a través de la señalización defectuosa de la leptina, efectos en el sistema nervioso central ${ }^{6-8}$. Además, los pacientes con diabetes tienen una mayor incidencia de enfermedad cardiovascular aterosclerótica, enfermedad arterial periférica y cerebrovascular. A su vez, la hipertensión y las anomalías del metabolismo de las lipoproteínas se encuentran a menudo en personas con diabetes 9 .

Para evitarlo, es preciso recalcar que la DMT2 está asociada a una gran variedad de factores de riesgo, entre los que se incluyen antecedentes familiares de diabetes, antecedentes de diabetes gestacional, 
inactividad física, glucemia de ayuno alterada, mala alimentación y obesidad ${ }^{6}$. En lo referente a la nutrición, un consumo elevado de algunos tipos de lípidos y azucares simples puede promover su desarroIlo, mientras que un consumo elevado de frutas y verduras puede representar un papel protector ${ }^{10-14}$.

\section{MODELOS ANIMALES DE DIABETES MELLITUS TIPO 2}

Como se ha descrito anteriormente, la DMT2 representa un grupo heterogéneo de trastornos, caracterizados por resistencia a la insulina y/o alteración de la secreción de insulina a causa de factores genéticos y del estilo de vida. Esto refleja la gran diversidad de modelos animales utilizados para su estudio. Se trata de herramientas esenciales para comprender la fisiopatología, las complicaciones microvasculares y macrovasculares, las acciones y el desarrollo de fármacos, y las influencias genéticas o ambientales que aumentan los riesgos de la DMT2, en formas que serían imposibles en humanos debido a problemas éticos ${ }^{15,16}$.

Entre todos los modelos experimentales disponibles, los roedores son los más utilizados debido a razones económicas, corto tiempo de generación e hiperglucemia hereditaria y/u obesidad presente en ciertas cepas ${ }^{17}$. Se clasifican según su mecanismo de producción, en modelos espontáneos o inducidos. Los modelos espontáneos incluyen animales en los que se ha detectado diabetes de manera espontánea o se han realizado una serie de cruces selectivos que favorecen cierto rasgo fenotípico de la DMT2 humana. Los modelos inducidos reproducen una o más manifestaciones clínicas de la DMT2 humana mediante una variedad de métodos, como la dieta o los métodos nutricionales, farmacológicos, quirúrgicos y genéticos ${ }^{18}$.
La decisión sobre el modelo a emplear para un experimento en particular es a menudo multifactorial. Idealmente, los experimentos deberían llevarse a cabo en varios modelos diferentes, teniendo en cuenta que ninguno de ellos refleja completamente la complejidad de la DMT2 humana y que se debe tener mucho cuidado al extrapolar los resultados a la clínica ${ }^{16}$. Con base en lo citado, el objetivo del presente trabajo es ofrecer una visión general sobre los diferentes tipos de modelos de roedores existentes para el estudio de la DMT2, con referencia a sus ventajas y desventajas, y evaluación de mecanismos subyacentes. Todo ello con el fin de conocer sus características y utilidad en el estudio de las complicaciones y los posibles usos en la investigación de DMT2, para así guiar a los investigadores en el ámbito de la diabetes hacia la selección más precisa y apropiada del modelo animal según los requisitos específicos del estudio.

\section{MÉTODOS}

La estrategia de búsqueda, la selección de publicaciones y los informes de los resultados de esta revisión se realizaron de acuerdo con las directrices de PRISMA (Preferred Reporting Items for Systematic review and Meta-Analysis protocols) ${ }^{19}$. Se realizó una búsqueda sistemática en una base de datos científica (PubMed) para la identificación de los estudios pertinentes. Se incluyeron artículos de revisión de texto completo publicados entre enero de 2011 y diciembre de 2018 en inglés y en español. La búsqueda se realizó utilizando los términos MeSH diabetes mellitus tipo 2 OR diabetes mellitus experimental AND murine OR modelos animales de enfermedad. Los criterios de inclusión de este estudio consistieron en revisiones de modelos roedores de DMT2. Los estudios se excluyeron cuando: 1) utilizaron animales distintos de los roedores, 2) se llevaron a cabo en humanos, 3) compararon la DMT2 con 
otras patologías sin centrarse en modelos animales, 4) se enfocaron en fármacos para el tratamiento de DMT2 o posibles dianas terapéuticas, y 5) se basaron en modelos animales específicos de complicaciones de la diabetes. Además, se realizó una exploración adicional de las listas de referencias en las revisiones y estudios relevantes para los fines de esta revisión, entre los que se incluyen artículos originales (Fig. 1).

\section{RESULTADOS}

Con la estrategia de búsqueda anteriormente citada se registraron, como se indica en la figura 1 , 98 artículos. De estos, 41 fueron excluidos debido a la lectura inicial de títulos. Tras la lectura de los resúmenes, se excluyeron un total de 39 artículos. De entre ellos, 15 por tratarse de modelos animales de otras enfermedades, tres por estar centrados en la relación entre DMT2 y funciones orgánicas, y 21 por evaluar diferentes parámetros farmacocinéticos de diferentes fármacos en la DMT2 o posibles dianas para futuras terapias. Dieciocho artículos de texto completo fueron evaluados para la elegibilidad; uno fue excluido porque solamente se enfocó en la base del fenotipado metabólico, uno por enfocarse en los genes y vías involucradas en DMT2 y objetivos para el descubrimiento de fármacos, y tres por centrarse en la identificación de genes relevantes para DMT2. Durante la revisión de texto completo, se incluyeron 18 artículos adicionales de la lista de referencias de los estudios que se estaban revisando. Finalmente, para esta revisión, se incluyeron un total de 31 estudios.

Los modelos animales de DMT2 incluyen modelos de resistencia a la insulina y/o modelos de daño en células $\beta$ pancreáticas, ya que la DMT2 se caracteriza por la resistencia a la insulina y la incapacidad de la células $\beta$ pancreáticas para compensar esta

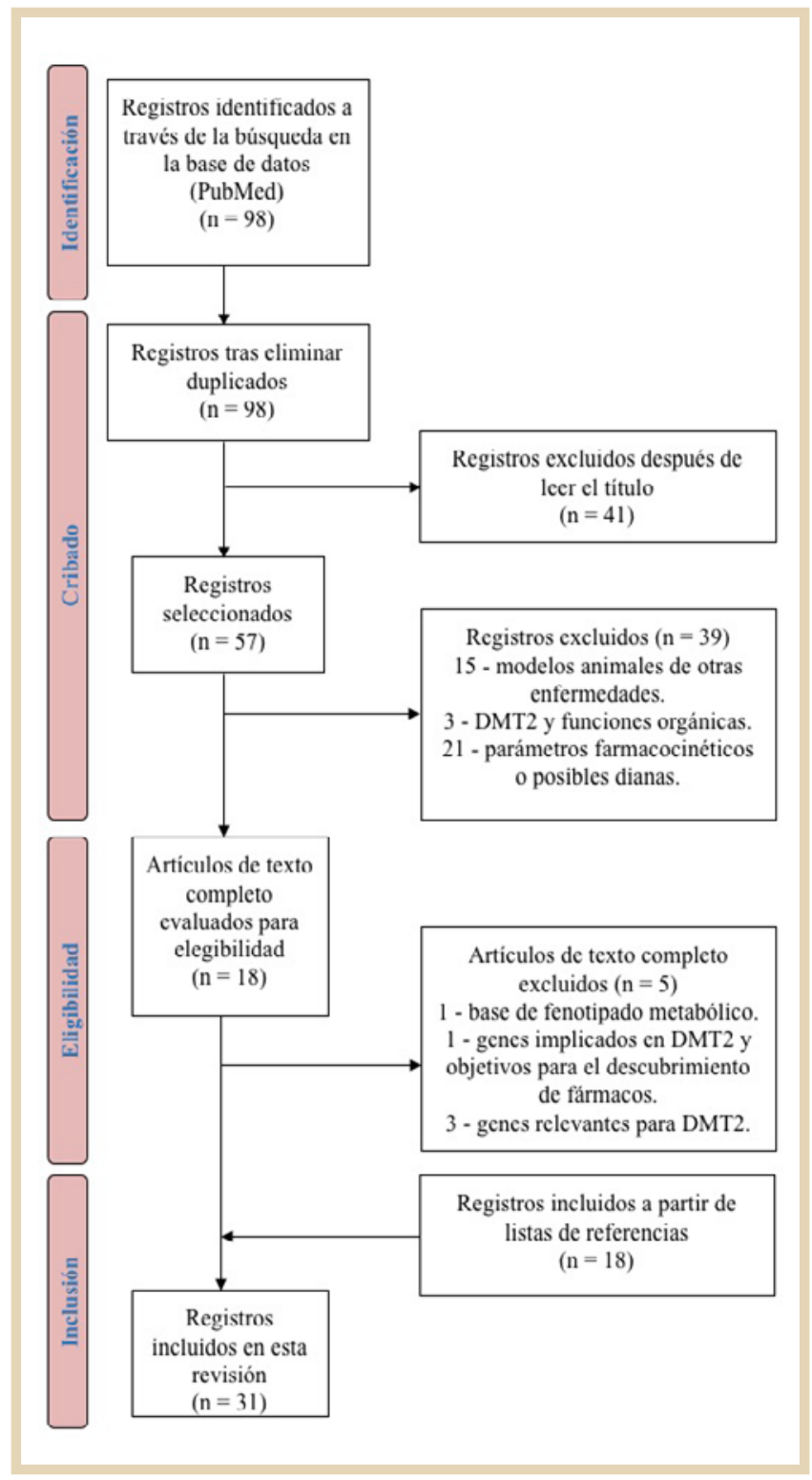

Figura 1. Diagrama de flujo de los estudios incluidos en esta revisión sistemática.

mayor demanda de insulina. Los modelos animales de DMT2 se pueden dividir en espontáneos/genéticos e inducidos, bien por medios dietéticos, farmacológicos, quirúrgicos o genéticos, que incluyen modelos transgénicos y knockout. En cada categoría, se subdividen en modelos con o sin obesidad, y en obesos espontáneos/genéticos, también se dividen según su genética en monogénicos o poligénicos, como se describe en la tabla 1. Cada 
TABLA 1. Modelos animales empleados en el estudio de la DMT2

\begin{tabular}{|c|c|c|c|c|}
\hline \multirow{3}{*}{$\begin{array}{l}\text { Modelos } \\
\text { Espontáneos/genéticos }\end{array}$} & \multicolumn{3}{|c|}{ Modelos obesos } & \multirow{3}{*}{$\begin{array}{l}\text { Modelos no obesos } \\
\text { Ratón AKITA } \\
\text { Rata GK } \\
\text { Rata Torii } \\
\text { Rata Cohen diabética }\end{array}$} \\
\hline & \multicolumn{2}{|l|}{ Monogénicos } & Poligénicos & \\
\hline & $\begin{array}{l}\text { Ratón ob/ob } \\
\text { Ratón } \mathrm{db} / \mathrm{db} \\
\text { Rata ZF } \\
\text { Rata ZDF }\end{array}$ & $\begin{array}{l}\text { Ratón } \mathrm{KK} \\
\text { Ratón KK/A } \\
\text { Ratón NZO } \\
\text { Ratón NONCNZO10 } \\
\text { Ratón TSOD } \\
\text { Ratón } \mathrm{M} 16\end{array}$ & $\begin{array}{l}\text { Ratón TallyHo } \\
\text { Rata OLETF } \\
\text { Rata WF } \\
\text { Rata SHR/N-cp } \\
\text { Rata JCR/LA-cp }\end{array}$ & \\
\hline Dietéticos (poligénicos) & \multicolumn{3}{|c|}{$\begin{array}{l}\text { Ratón } \mathrm{C} 57 / \mathrm{BL} 6 \mathrm{~J} \\
\text { Ratón Spiny } \\
\text { Rata Sand }\end{array}$} & - \\
\hline Manipulación farmacológica & \multicolumn{3}{|c|}{ Administración de GTG en ratones } & $\begin{array}{l}\text { Administración de ALX o STZ } \\
\text { en ratas } \\
\text { Administración neonatal } \\
\text { de STZ en ratas }\end{array}$ \\
\hline Manipulación quirúrgica & \multicolumn{3}{|c|}{ Rata con lesiones del hipotálamo ventromedial } & $\begin{array}{l}\text { Ratas pancreatomizadas } \\
\text { parciales }\end{array}$ \\
\hline Manipulación genética & \multicolumn{3}{|c|}{$\begin{array}{l}\text { Ratón knockout receptor adrenérgico } \beta_{3} \\
\text { Ratón knockout UCP1 } \\
\text { Ratón knockout BDNF }\end{array}$} & $\begin{array}{l}\text { Ratón knockout IR } \\
\text { Ratón knockout IRS-1, IRS-2 } \\
\text { Ratón knockout GLUT-4 } \\
\text { Ratón knockout PPAR- } \gamma \\
\text { Ratón transgénico hIAPP }\end{array}$ \\
\hline
\end{tabular}

Ob: obeso; db: diabético; ZF: Zucker obesa; ZDF: Zucker diabética y obesa; KK: Kuo Kondo; KK/AY: KK amarillo y obeso; NZO: obeso de Nueva Zelanda; NONcNZO10: ratón NZO junto con ratón no obeso-no diabético; TSOD: Tsumara Suzuki obeso y diabético; OLETF: Otuska Long Evans Tokushima obesa; WF: Wistar obesa; SHR/N-cp: rata espontáneamente hipertensa/NIH corpulenta; GK: Goto-Kakizaki; GTG: tioglucosa de oro; ALX: aloxán; STZ: estreptomicina; UCP1: proteína desacoplante del tejido adiposo pardo; BDNF: factor neurotrófico derivado del cerebro; IR: receptor insulina; IRS: sustrato para el receptor de insulina; GLUT-4: transportador de glucosa 4; PPAR- $\gamma$ : receptor activado por proliferadores de peroxisomas gamma; hIAPP: proteína polipéptido amiloide de los islotes pancreáticos humanos.

modelo presenta características diferentes, que determinan sus posibles usos en el estudio de las complicaciones de DMT2 y la investigación (Tabla 2).

\section{DISCUSIÓN}

Esta revisión identificó 31 publicaciones que estudian los diferentes modelos empleados en el estudio de la DMT2. El artículo más antiguo se publicó en 2011, mientras que el más reciente en 2018. Una cantidad creciente de publicaciones sobre este tema muestra el interés de la comunidad científica en mejorar y descubrir modelos animales que puedan utilizarse como herramienta para prevenir o tratar la DMT2. Los modelos difieren significativamente, por lo que, dependiendo de la finalidad de la investigación, deben considerarse las ventajas y desventajas de cada uno de ellos.

Dentro de los modelos espontáneos monogénicos, se encuentran el ratón obeso ob/ob, el ratón diabético db/db, la rata Zucker obesa (ZF) y la rata Zucker diabética y obesa (ZFD). El ratón ob/ob, también llamado Lep ${ }^{\mathrm{ob} / \mathrm{ob}}$, presenta una mutación (ob) en el gen de la leptina, con la consiguiente deficiencia de esta al no poder unirse a sus receptores. El ratón $\mathrm{db} / \mathrm{db}$ o Lepr ${ }^{\mathrm{db} / \mathrm{db}}$ presenta una mutación ( $\mathrm{db}$ ) en el receptor de leptina, que le confiere una menor actividad. La rata ZF presenta una mutación ( $\mathrm{fa}$ ) en el receptor de la leptina, mientras que la rata ZFD surgió de la cría selectiva de ratas $Z^{23,51}$. En comparación con los modelos poligénicos, los modelos monogénicos pueden ser útiles para comprender cómo se desarrolla la resistencia a la insulina en un fenotipo obeso ${ }^{51}$. 
TABLA 2. Características metabólicas, complicaciones clínicas y usos de los principales modelos empleados en el estudio de la DMT2

\begin{tabular}{|c|c|c|c|c|}
\hline Modelo & Características metabólicas & Complicaciones clínicas & Usos & Referencias \\
\hline \multicolumn{5}{|c|}{ Espontáneos/genéticos } \\
\hline Ratón ob/ob & $\begin{array}{l}\text { Hiperfagia, obesidad, } \\
\text { hiperlipidemia (HDL), } \\
\text { hiperglucemia transitoria } \\
\text { leve, hiperinsulinemia, } \\
\text { resistencia a la insulina, } \\
\text { hipertrofia e hiperplasia } \\
\text { de células } \beta \text { pancreáticas }\end{array}$ & $\begin{array}{l}\text { Neuropatía periférica } \\
\text { diabética }\end{array}$ & $\begin{array}{l}\text { Desarrollo de compuestos } \\
\text { antiobesidad, } \\
\text { antidiabéticos y agentes } \\
\text { que aumenten la } \\
\text { sensibilidad a la insulina }\end{array}$ & $\begin{array}{c}20,21,22,23,24,25 \\
26,27,28,29\end{array}$ \\
\hline Ratón db/db & $\begin{array}{l}\text { Hiperfagia, obesidad, } \\
\text { hiperlipidemia (HDL), } \\
\text { hiperglucemia, } \\
\text { hiperinsulinemia, } \\
\text { resistencia a la insulina, } \\
\text { disfunción de células } \beta \\
\text { pancreáticas }\end{array}$ & $\begin{array}{l}\text { Neuropatía periférica } \\
\text { diabética } \\
\text { Nefropatía diabética } \\
\text { Retinopatía diabética }\end{array}$ & $\begin{array}{l}\text { Tratamientos para mejorar } \\
\text { la función de las células } \beta \\
\text { pancreáticas. Desarrollo } \\
\text { de análogos de insulina, } \\
\text { compuestos que } \\
\text { aumenten la sensibilidad } \\
\text { a la insulina y agentes } \\
\text { antiobesidad }\end{array}$ & $\begin{array}{l}20,21,22,23,24,25 \\
26,27\end{array}$ \\
\hline Rata ZF & $\begin{array}{l}\text { Hiperfagia, obesidad, } \\
\text { hiperlipidemia (LDL, } \\
\text { HDL), leve intolerancia } \\
\text { a la glucosa, leve } \\
\text { hiperglucemia, } \\
\text { hiperinsulinemia, } \\
\text { resistencia a la insulina e } \\
\text { hipertensión }\end{array}$ & $\begin{array}{l}\text { Neuropatía periférica } \\
\text { diabética } \\
\text { Nefropatía diabética }\end{array}$ & $\begin{array}{l}\text { Desarrollo de agentes para } \\
\text { el tratamiento de } \\
\text { obesidad y resistencia a la } \\
\text { insulina } \\
\text { Estudio de hipertensión } \\
\text { y aterosclerosis }\end{array}$ & $20,21,22,23,30$ \\
\hline Rata ZDF & $\begin{array}{l}\text { Hiperfagia, obesidad, } \\
\text { hiperlipidemia (LDL, } \\
\text { HDL), hiperglucemia en } \\
\text { machos, hiperinsulinemia, } \\
\text { resistencia a la insulina, } \\
\text { disfunción de células } \beta \\
\text { pancreáticas }\end{array}$ & $\begin{array}{l}\text { Neuropatía periférica } \\
\text { diabética } \\
\text { Nefropatía diabética } \\
\text { Retinopatía diabética }\end{array}$ & $\begin{array}{l}\text { Desarrollo de agentes para } \\
\text { el tratamiento de la } \\
\text { obesidad, diabetes y } \\
\text { resistencia a la insulina } \\
\text { Estudio de complicaciones } \\
\text { diabéticas }\end{array}$ & $\begin{array}{l}20,21,22,23,25,26 \\
27,30\end{array}$ \\
\hline Ratón KK & $\begin{array}{l}\text { Hiperfagia, obesidad } \\
\text { moderada, intolerancia a } \\
\text { la glucosa, hiperglucemia, } \\
\text { hiperinsulinemia, } \\
\text { resistencia a la insulina, } \\
\text { hipertrofia pancreática }\end{array}$ & $\begin{array}{l}\text { Neuropatía periférica } \\
\text { diabética } \\
\text { Nefropatía diabética } \\
\text { Retinopatía diabética }\end{array}$ & $\begin{array}{l}\text { Desarrollo de agentes para } \\
\text { el tratamiento de la } \\
\text { obesidad, diabetes y } \\
\text { resistencia a la insulina } \\
\text { Estudio de complicaciones } \\
\text { diabéticas }\end{array}$ & $21,22,27,32$ \\
\hline Ratón $K K / A^{Y}$ & $\begin{array}{l}\text { Mismas manifestaciones } \\
\text { que KK, pero mucho más } \\
\text { pronunciadas }\end{array}$ & $\begin{array}{l}\text { Neuropatía periférica } \\
\text { diabética } \\
\text { Nefropatía diabética } \\
\text { Retinopatía diabética }\end{array}$ & $\begin{array}{l}\text { Desarrollo de agentes } \\
\text { antidiabéticos } \\
\text { e hipoglucemiantes } \\
\text { Estudio de las } \\
\text { complicaciones diabéticas }\end{array}$ & $20,21,22,27$ \\
\hline Ratón NZO & $\begin{array}{l}\text { Hiperfagia, obesidad, } \\
\text { intolerancia a la glucosa, } \\
\text { hiperglucemia, } \\
\text { hiperinsulinemia, } \\
\text { resistencia a la insulina, } \\
\text { hiperplasia e hipertrofia } \\
\text { pancreática, disfunción } \\
\text { de células } \beta \text { pancreáticas }\end{array}$ & Nefropatía diabética & $\begin{array}{l}\text { Estudio de cualquier } \\
\text { agente que muestre } \\
\text { relación entre } \\
\text { autoinmunidad, obesidad } \\
\text { y diabetes }\end{array}$ & $20,21,22,27,33,34$ \\
\hline Ratón NONc/NZO10 & $\begin{array}{l}\text { Obesidad, hiperglucemia, } \\
\text { hiperinsulinemia, } \\
\text { resistencia a la insulina, } \\
\text { hipertrofia y disfunción } \\
\text { de células } \beta \text { pancreáticas }\end{array}$ & Nefropatía diabética & $\begin{array}{l}\text { Estudio del término } \\
\text { «Diabesidad», referente a } \\
\text { la diabetes que ocurre en } \\
\text { el contexto de la } \\
\text { obesidad }\end{array}$ & $21,22,34$ \\
\hline
\end{tabular}


TABLA 2. Características metabólicas, complicaciones clínicas y usos de los principales modelos empleados en el estudio de la DMT2 (continuación)

\begin{tabular}{|c|c|c|c|c|}
\hline Modelo & Características metabólicas & Complicaciones clínicas & Usos & Referencias \\
\hline Ratón TSOD & $\begin{array}{l}\text { Obesidad, polidipsia, } \\
\text { poliuria, hiperglucemia, } \\
\text { hiperinsulinemia, } \\
\text { resistencia a la insulina, } \\
\text { hipertrofia de células } \beta \\
\text { pancreáticas }\end{array}$ & $\begin{array}{l}\text { Neuropatía periférica } \\
\text { diabética } \\
\text { Nefropatía diabética }\end{array}$ & $\begin{array}{l}\text { Estudio de complicaciones } \\
\text { diabéticas }\end{array}$ & 21,35 \\
\hline Ratón M16 & $\begin{array}{l}\text { Hiperfagia, obesidad } \\
\text { moderada, } \\
\text { hipercolesterolemia, } \\
\text { hiperglucemia e } \\
\text { hiperinsulinemia }\end{array}$ & No se han observado & $\begin{array}{l}\text { Descubrimiento de genes y } \\
\text { regulación de la vía que } \\
\text { controla la aparición } \\
\text { temprana de obesidad } \\
\text { poligénica y fenotipos } \\
\text { presentes en la DMT2 }\end{array}$ & 36 \\
\hline Ratón TallyHo & $\begin{array}{l}\text { Obesidad, } \\
\text { hipertrigliceridemia, } \\
\text { intolerancia a la glucosa, } \\
\text { hiperglucemia, } \\
\text { hiperinsulinemia, } \\
\text { hipertrofia e hiperplasia } \\
\text { pancreática, disfunción } \\
\text { de células } \beta \text { pancreáticas }\end{array}$ & Nefropatía diabética & $\begin{array}{l}\text { Estudio de la base genética } \\
\text { de la DMT2 } \\
\text { Desarrollo de agentes } \\
\text { antiobesidad y } \\
\text { antidiabéticos }\end{array}$ & $20,21,22,24,34,37$ \\
\hline Rata OLETF & $\begin{array}{l}\text { Hiperfagia, obesidad leve, } \\
\text { hipertrigliceridemia, } \\
\text { hipercolesterolemia, } \\
\text { hiperglucemia, } \\
\text { hiperinsulinemia, } \\
\text { resistencia a la insulina, } \\
\text { hiperplasia de células } \beta \\
\text { pancreáticas }\end{array}$ & $\begin{array}{l}\text { Neuropatía periférica } \\
\text { diabética } \\
\text { Nefropatía diabética } \\
\text { Retinopatía diabética }\end{array}$ & $\begin{array}{l}\text { Desarrollo de agentes } \\
\text { antidiabéticos y análogos } \\
\text { de insulina }\end{array}$ & $\begin{array}{l}20,21,22,25,26,27 \\
\quad 38,39\end{array}$ \\
\hline Rata WF & $\begin{array}{l}\text { Hiperfagia, obesidad, } \\
\text { hipertrigliceridemia, } \\
\text { intolerancia a la glucosa, } \\
\text { hiperinsulinemia }\end{array}$ & $\begin{array}{l}\text { Neuropatía periférica } \\
\text { diabética }\end{array}$ & $\begin{array}{l}\text { Estudio de la etiología y } \\
\text { patología de la DMT2 } \\
\text { humana y desarrollo de } \\
\text { agentes antidiabéticos }\end{array}$ & 21,27 \\
\hline $\mathrm{SHR} / \mathrm{N}-\mathrm{cP}$ & $\begin{array}{l}\text { Hiperfagia, obesidad, } \\
\text { hiperlipidemia (LDL, } \\
\text { HDL), hiperglucemia } \\
\text { pospandrial, } \\
\text { hiperinsulinemia, } \\
\text { resistencia a la insulina }\end{array}$ & $\begin{array}{l}\text { Nefropatía diabética } \\
\text { Retinopatía diabética }\end{array}$ & $\begin{array}{l}\text { Estudio de la DMT2 } \\
\text { asociada a obesidad y } \\
\text { estudio de los efectos de } \\
\text { las dietas con } \\
\text { carbohidratos en el } \\
\text { desarrollo de la diabetes }\end{array}$ & $21,23,26,27$ \\
\hline$J C R / L A-C P$ & $\begin{array}{l}\text { Hiperfagia, obesidad, } \\
\text { hiperlipidemia (VLDL), } \\
\text { hiperglucemia moderada, } \\
\text { hiperinsulinemia, } \\
\text { resistencia a la insulina }\end{array}$ & Nefropatía diabética & Estudio de aterosclerosis & $21,23,27$ \\
\hline Rata GK & $\begin{array}{l}\text { Leve hiperglucemia, } \\
\text { deficiente secreción de } \\
\text { insulina tras sobrecarga } \\
\text { de glucosa, leve } \\
\text { hiperinsulinemia, leve } \\
\text { resistencia a la insulina, } \\
\text { hipoplasia pancreática, } \\
\text { menor número y } \\
\text { disfunción de células } \beta \\
\text { pancreáticas }\end{array}$ & $\begin{array}{l}\text { Neuropatía periférica } \\
\text { diabética } \\
\text { Nefropatía diabética } \\
\text { Retinopatía diabética }\end{array}$ & $\begin{array}{l}\text { Estudio de los mecanismos } \\
\text { de destrucción y pérdida } \\
\text { de células } \beta \text { pancreáticas } \\
\text { Tratamientos para mejorar } \\
\text { la supervivencia y función } \\
\text { de las células } \beta \\
\text { pancreáticas }\end{array}$ & $\begin{array}{l}20,21,22,25,26,27, \\
31,40\end{array}$ \\
\hline
\end{tabular}


TABLA 2. Características metabólicas, complicaciones clínicas y usos de los principales modelos empleados en el estudio de la DMT2 (continuación)

\begin{tabular}{|c|c|c|c|c|}
\hline Modelo & Características metabólicas & Complicaciones clínicas & Usos & Referencias \\
\hline Rata Torii & $\begin{array}{l}\text { Hipertrigliceridemia, } \\
\text { intolerancia a la glucosa } \\
\text { hiperglucemia, } \\
\text { hipoinsulinemia, atrofia y } \\
\text { disfunción de células } \beta \\
\text { pancreáticas }\end{array}$ & $\begin{array}{l}\text { Neuropatía periférica } \\
\text { diabética } \\
\text { Nefropatía diabética } \\
\text { Retinopatía diabética }\end{array}$ & $\begin{array}{l}\text { Desarrollo de fármacos } \\
\text { antidiabéticos y fármacos } \\
\text { y/o terapia génica para } \\
\text { las complicaciones } \\
\text { diabéticas } \\
\text { Estudio de la depleción en } \\
\text { la secreción de insulina e } \\
\text { intolerancia a la glucosa }\end{array}$ & 41 \\
\hline Rata Cohen diabética & $\begin{array}{l}\text { Exhibe DMT2 inducida por } \\
\text { la dieta, con } \\
\text { características propias de } \\
\text { la DMT2 humana, al } \\
\text { expresar susceptibilidad } \\
\text { genética a una dieta rica } \\
\text { en carbohidratos } \\
\text { Hiperglucemia, } \\
\text { hiperinsulinemia, } \\
\text { resistencia a la insulina, } \\
\text { hipoinsulinemia }\end{array}$ & $\begin{array}{l}\text { Nefropatía diabética } \\
\text { Retinopatía diabética }\end{array}$ & $\begin{array}{l}\text { Estudiar la interacción } \\
\text { entre los factores } \\
\text { ambientales y } \\
\text { nutricionales y la } \\
\text { susceptibilidad genética } \\
\text { para el desarrollo de } \\
\text { DMT2 }\end{array}$ & 21 \\
\hline \multicolumn{5}{|l|}{ Dietéticos } \\
\hline $\begin{array}{l}\text { Ratón } \\
\text { C57/BL 6J }\end{array}$ & $\begin{array}{l}\text { Alimentados con una dieta } \\
\text { alta en grasa desarrollan } \\
\text { obesidad, dislipidemia, } \\
\text { intolerancia a la glucosa, } \\
\text { leve hiperglucemia, } \\
\text { hiperinsulinemia, } \\
\text { resistencia a la insulina }\end{array}$ & $\begin{array}{l}\text { Neuropatía periférica } \\
\text { diabética } \\
\text { Nefropatía diabética }\end{array}$ & $\begin{array}{l}\text { Estudio de factores } \\
\text { controlables en estadios } \\
\text { previos al desarrollo de la } \\
\text { enfermedad, así como } \\
\text { agentes capaces de } \\
\text { reducir la progresión o } \\
\text { revertir el avance de la } \\
\text { enfermedad }\end{array}$ & $21,22,24,42,44,45$ \\
\hline Ratón Spiny & $\begin{array}{l}\text { Desarrolla DMT2 de un } \\
\text { modo diferente a la } \\
\text { enfermedad humana. } \\
\text { Alimentado con una dieta } \\
\text { alta en grasa, presenta } \\
\text { obesidad, leve } \\
\text { hiperglucemia, } \\
\text { hiperinsulinemia, } \\
\text { hipertrofia, proliferación, } \\
\text { degeneración y rotura } \\
\text { espontánea de los islotes } \\
\text { pancreáticos, } \\
\text { hipoinsulinemia }\end{array}$ & No se han observado & $\begin{array}{l}\text { Estudio de la diabetes } \\
\text { inducida mediante la } \\
\text { dieta, por tener esta una } \\
\text { alta prevalencia en } \\
\text { humanos }\end{array}$ & 22,47 \\
\hline Rata Sand & $\begin{array}{l}\text { Alimentadas con una dieta } \\
\text { similar a la Western, } \\
\text { desarrollan hiperfagia, } \\
\text { obesidad, leve } \\
\text { hiperglucemia, } \\
\text { hiperinsulinemia, } \\
\text { resistencia a la insulina, } \\
\text { degeneración de células } \beta \\
\text { pancreáticas, } \\
\text { hipoinsulinemia }\end{array}$ & $\begin{array}{l}\text { Neuropatía periférica } \\
\text { diabética } \\
\text { Nefropatía diabética } \\
\text { Retinopatía diabética }\end{array}$ & $\begin{array}{l}\text { Estudio de los efectos de la } \\
\text { dieta y el ejercicio en el } \\
\text { desarrollo de la DMT2 }\end{array}$ & $21,26,48$ \\
\hline
\end{tabular}


TABLA 2. Características metabólicas, complicaciones clínicas y usos de los principales modelos empleados en el estudio de la DMT2 (continuación)

\begin{tabular}{|c|c|c|c|c|}
\hline Modelo & Características metabólicas & Complicaciones clínicas & Usos & Referencias \\
\hline \multicolumn{5}{|c|}{ Manipulación farmacológica } \\
\hline $\begin{array}{l}\text { Administración } \\
\text { de GTG } \\
\text { en ratones }\end{array}$ & $\begin{array}{l}\text { Hiperfagia, obesidad, } \\
\text { hiperlipidemia, } \\
\text { hiperglucemia, } \\
\text { hiperinsulinemia, } \\
\text { resistencia a la insulina, } \\
\text { hipoinsulinemia }\end{array}$ & $\begin{array}{l}\text { Diferentes según el } \\
\text { modelo empleado }\end{array}$ & $\begin{array}{l}\text { Estudio del papel de los } \\
\text { factores ambientales en } \\
\text { los procesos de } \\
\text { destrucción pancreática }\end{array}$ & 49 \\
\hline $\begin{array}{l}\text { Administración } \\
\text { de } A L X \\
\text { en ratas }\end{array}$ & $\begin{array}{l}\text { Primero desarrollan } \\
\text { hipoglucemia transitoria, } \\
\text { hiperinsulinemia, después } \\
\text { hiperglucemia, } \\
\text { hipoinsulinemia, toxicidad } \\
\text { y destrucción de células } \beta \\
\text { pancreáticas y finalmente } \\
\text { hipoglucemia }\end{array}$ & $\begin{array}{l}\text { Diferentes según el } \\
\text { modelo empleado }\end{array}$ & $\begin{array}{l}\text { Selección de análogos de } \\
\text { insulina y otros } \\
\text { compuestos } \\
\text { hipoglucemiantes }\end{array}$ & $20,24,26,31,46$ \\
\hline $\begin{array}{l}\text { Administración } \\
\text { de STZ } \\
\text { en ratas }\end{array}$ & $\begin{array}{l}\text { Hiperglucemia, } \\
\text { hipoinsulinemia, muerte } \\
\text { de células } \beta \text { pancreáticas }\end{array}$ & $\begin{array}{l}\text { Diferentes según el } \\
\text { modelo empleado }\end{array}$ & $\begin{array}{l}\text { Selección de análogos de } \\
\text { insulina y otros } \\
\text { compuestos } \\
\text { hipoglucemiantes }\end{array}$ & $\begin{array}{l}21,24,25,27,31,42 \\
46\end{array}$ \\
\hline $\begin{array}{l}\text { Administración neonatal } \\
\text { de STZ en ratas }\end{array}$ & $\begin{array}{l}\text { Hiperglucemia, seguida de } \\
\text { regeneración de las } \\
\text { células } \beta \text { pancreáticas y } \\
\text { normoglucemia, para } \\
\text { finalmente, volver a } \\
\text { acabar desarrollando } \\
\text { hiperglucemia, disfunción } \\
\text { y disminución de células } \beta \\
\text { pancreáticas }\end{array}$ & $\begin{array}{l}\text { Diferentes según el } \\
\text { modelo empleado }\end{array}$ & $\begin{array}{l}\text { Estudios cuyo objetivo es } \\
\text { estimular la regeneración } \\
\text { de las células } \beta \\
\text { pancreáticas tras una } \\
\text { lesión de estas }\end{array}$ & $22,26,46$ \\
\hline \multicolumn{5}{|l|}{ Manipulación quirúrgica } \\
\hline $\begin{array}{l}\text { Ratas pancreatomizadas } \\
\text { parciales }\end{array}$ & $\begin{array}{l}\text { Hiperglucemia moderada, } \\
\text { sin significativos cambios } \\
\text { en peso corporal o } \\
\text { niveles de insulinemia, } \\
\text { deterioro en la secreción } \\
\text { de insulina y capacidad de } \\
\text { regeneración de células } \beta \\
\text { pancreáticas }\end{array}$ & $\begin{array}{l}\text { Diferentes según el } \\
\text { modelo empleado }\end{array}$ & $\begin{array}{l}\text { Estudio de la reducción de } \\
\text { células } \beta \text { pancreáticas, así } \\
\text { como investigación sobre } \\
\text { trasplantes de islotes } \\
\text { pancreáticos y } \\
\text { regeneración de estos }\end{array}$ & 22,46 \\
\hline \multicolumn{5}{|l|}{ Manipulación genética } \\
\hline $\begin{array}{l}\text { Ratón knockout receptor } \\
\text { adrenérgico } \beta_{3}\end{array}$ & $\begin{array}{l}\text { Obesidad, resistencia a la } \\
\text { insulina, temprano inicio } \\
\text { de DMT2 }\end{array}$ & No se han observado & $\begin{array}{l}\text { Desarrollo de agonistas } \\
\text { selectivos para los } \\
\text { receptores } \beta_{3} \\
\text { adrenérgicos como } \\
\text { posibles fármacos } \\
\text { antiobesidad }\end{array}$ & 24 \\
\hline Ratón knockout BDNF & $\begin{array}{l}\text { Hiperfagia, obesidad, } \\
\text { hiperglucemia, } \\
\text { hiperinsulinemia, } \\
\text { resistencia a la insulina }\end{array}$ & No se han observado & $\begin{array}{l}\text { Desarrollo de agentes } \\
\text { terapéuticos para } \\
\text { prevenir y tratar } \\
\text { anomalías en la } \\
\text { regulación del peso } \\
\text { corporal y el metabolismo } \\
\text { energético }\end{array}$ & 21,24 \\
\hline
\end{tabular}


TABLA 2. Características metabólicas, complicaciones clínicas y usos de los principales modelos empleados en el estudio de la DMT2 (continuación)

\begin{tabular}{|c|c|c|c|c|}
\hline Modelo & Características metabólicas & Complicaciones clínicas & Usos & Referencias \\
\hline Ratón knockout IR & $\begin{array}{l}\text { Homocigóticos: } \\
\text { hiperglucemia, } \\
\text { cetoacidosis diabética, } \\
\text { muerte } \\
\text { Heterocigóticos: solo el 10\% } \\
\text { desarrolla diabetes }\end{array}$ & No se han observado & $\begin{array}{l}\text { Estudio de las } \\
\text { consecuencias de la falta } \\
\text { del gen receptor de } \\
\text { insulina }\end{array}$ & 50 \\
\hline Ratón knockout IRS-1 & $\begin{array}{l}\text { Hipertrigliceridemia, leve } \\
\text { intolerancia a la glucosa, } \\
\text { hiperinsulinemia, leve } \\
\text { resistencia a la insulina, } \\
\text { hiperplasia de células } \beta \\
\text { pancreáticas }\end{array}$ & No se han observado & $\begin{array}{l}\text { Investigar el papel de IRS-1 } \\
\text { en diferentes órganos }\end{array}$ & $21,25,26,50$ \\
\hline Ratón knockout IRS-2 & $\begin{array}{l}\text { Resistencia a la insulina, } \\
\text { reducción de células } \beta \\
\text { pancreáticas }\end{array}$ & No se han observado & $\begin{array}{l}\text { Estudio de la función y } \\
\text { desarrollo de las células } \beta \\
\text { pancreáticas }\end{array}$ & $21,25,26,50$ \\
\hline Ratón knockout GLUT-4 & $\begin{array}{l}\text { Hiperglucemia, } \\
\text { hiperinsulinemia, } \\
\text { resistencia a la insulina }\end{array}$ & No se han observado & $\begin{array}{l}\text { Estudio desarrollo de la } \\
\text { DMT2 sin complicaciones } \\
\text { relacionadas con } \\
\text { obesidad }\end{array}$ & $21,24,26,50$ \\
\hline Ratón transgénico hIAPP & $\begin{array}{l}\text { Deposición proteína } \\
\text { amiloidea en el páncreas, } \\
\text { toxicidad de células } \beta \\
\text { pancreáticas }\end{array}$ & No se han observado & $\begin{array}{l}\text { Tratamientos para evitar la } \\
\text { deposición de proteína } \\
\text { amiloidea, así como para } \\
\text { mejorar la supervivencia } \\
\text { de las células } \beta \\
\text { pancreáticas }\end{array}$ & 22 \\
\hline
\end{tabular}

Ob: obeso; db: diabético; ZF: Zucker obesa; ZDF: Zucker diabética y obesa; KK: Kuo Kondo; KK/A: KK amarillo y obeso; NZO: obeso de Nueva Zelanda; NONcNZO10: ratón NZO junto con ratón no obeso-no diabético; TSOD: Tsumara Suzuki obeso y diabético; OLETF: Otuska Long Evans Tokushima obesa; WF: Wistar obesa;

SHR/N-cp: rata espontáneamente hipertensa/NIH corpulenta; GK: Goto-Kakizaki; GTG: tioglucosa de oro; ALX: aloxán; STZ: estreptomicina; UCP1: proteína desacoplante del tejido adiposo pardo; BDNF: factor neurotrófico derivado del cerebro; IR: receptor insulina; IRS: sustrato para el receptor de insulina; GLUT-4: transportador de glucosa 4; PPAR- $\gamma$ : receptor activado por proliferadores de peroxisomas gamma; hIAPP: proteína polipéptido amiloide de los islotes pancreáticos humanos; HDL: lipoproteínas de alta densidad; LDL: lipoproteínas de baja densidad; VLDL: lipoproteínas de muy baja densidad; DMT2: diabetes mellitus tipo 2.

Los modelos espontáneos poligénicos incluyen el ratón Kuo Kondo (KK), el ratón KK amarillo y obeso $\left(K K / A^{\gamma}\right)$, el ratón obeso de Nueva Zelanda (NZO), el ratón NZO junto con ratón no obeso-no diabético (NONc/NZO10), el ratón Tsumara Suzuki obeso y diabético (TSOD), el ratón M16, el ratón TallyHo, la rata Otuska Long Evans Tokushima obesa (OLETF), la rata Wistar obesa (WF), la rata espontáneamente hipertensa/NIH corpulenta (SHR/N-cP), la rata JCR/ LA-cp, la rata Goto-Kakizaki (GK), la rata Torri y la rata Cohen diabética. El ratón KK fue desarrollado mediante la cría selectiva de ratones de gran tamaño corporal ${ }^{52}$, mientras que el ratón $K K / A^{\gamma}$ fue generado mediante la adición del gen letal $A^{\gamma}$ a los ratones $\mathrm{KK}^{53}$. El ratón NZO se desarrolló mediante la cría selectiva de ratones obesos derivados de un par de ratones Agouti54, mientras que el ratón NONc/ NZO10 surgió de cruzar ratones NZO con ratones no obesos y no diabéticos ${ }^{55}$. El ratón TSOD fue desarrollado mediante la cría selectiva de ratones de la cepa Deutschland, Denken, and Yoken (DDY) ${ }^{56}$. El ratón M16 se creó a través de la selección a largo plazo de ratones ICR (Instituto de Investigación del Cáncer), una cepa de ratones albinos, desarrollados para ganar peso corporal57. El ratón TallyHo deriva de ratones Theiler Original con poliuria e hiperglucemia57. La rata OLETF carece del receptor de la colecistoquinina, un péptido que juega un papel importante en los mecanismos de saciedad, y surgió de la cría selectiva de las ratas Long Evans empleadas para el 
estudio de la obesidad inducida mediante la dieta ${ }^{38}$. La rata WF fue desarrollada a partir de la cría selectiva de ratas Wistar Kyoto, a las cuales se les transfirió el gen fa de la rata ZF, presentando por tanto una actividad reducida del receptor de la leptina ${ }^{58}$. Al igual que en el caso anterior, tanto la rata SHR/N-cp como la rata JCR/LA-cp presentan una mutación en el gen cp que causa la ausencia de una porción del receptor de la leptina y una disminución de la actividad de este ${ }^{23}$. La rata GK fue desarrollada a partir de una colonia de ratas Wistar, mediante la selección de ratas con hiperglucemia ${ }^{8}$. La rata Torri fue desarrollada a partir de la cepa Sprague Dawley ${ }^{41}$. Por último, la rata Cohen diabética, un modelo derivado de modelos de DMT2 inducidos nutricionalmente, tras la administración de una dieta alta en sacarosa y pobre en cobre durante dos meses ${ }^{59}$.

Teniendo en cuenta los modelos espontáneos anteriormente citados podríamos destacar algunas ventajas como el desarrollo espontáneo de la enfermedad, con características similares a la DMT2 humana, la mínima variabilidad observada en cuestión de resultados en los estudios realizados y el requerimiento de un número pequeño de animales para llevarlos a cabo, así como el buen control de factores ambientales, debido a que los genéticos ya están determinados ${ }^{60}$. Concretamente, en el ratón $\mathrm{KK}$, un estudio revela que la severidad de la diabetes depende estrictamente del peso corporal. Para ello, se evaluó la acción diabetogénica de la obesidad en la cepa KK y en la cepa C57/BL 6 mediante una dieta rica en grasas y mediante la administración de tioglucosa de oro (GTG). Solamente desarroIló hiperglucemia severa y resistencia a la insulina el ratón KK. Así, concluyeron que la diferencia entre ambas cepas era debida a factores genéticos ${ }^{32}$.

Como desventajas, podrían señalarse que el desarrollo de la DMT2 está muy determinado genéticamente, a diferencia de lo que ocurre en humanos, en los cuales la genética tiene una importancia menor respecto a los factores ambientales. A su vez, sus sofisticadas condiciones de mantenimiento y su corta esperanza de vida limitan la dimensión de los estudios realizados ${ }^{60}$.

Los modelos dietéticos incluyen el ratón C57/BL 6J, el ratón Spiny y la rata Sand.

El ratón $\mathrm{C} 57 / \mathrm{BL} 6 \mathrm{~J}$ es alimentado con una dieta alta en grasa, de tal manera que la dieta basal se sustituye por una dieta en la cual el porcentaje de grasa asciende hasta llegar al 58\% de la energía total administrada ${ }^{22}$. El ratón Spiny desarrolla diabetes cuando se le administra una dieta suplementada con semillas ricas en grasa47. Por último, la rata Sand es propensa a desarrollar diabetes, probabilidad que se ve aumentada al ser alimentada con una dieta alta en calorías ${ }^{48}$.

Como ventajas de los modelos dietéticos se incluyen que son modelos poligénicos y que, además, el desarrollo de la DMT2 está asociado con la obesidad, debido a unos hábitos dietéticos no saludables, como ocurre en humanos. Otra ventaja podría ser que para su desarrollo no son necesarios compuestos capaces de provocar toxicidad en otros órganos, como ocurre en los modelos inducidos farmacológicamente ${ }^{21}$. Como desventajas podrían señalarse que los animales requieren largos periodos de tratamiento dietético para llegar a las condiciones óptimas de estudio y que no presentan una hiperglucemia significativa, haciéndolos no adecuados para estudios en los que se determinan las concentraciones sanguíneas de glucosa en el desarrollo de compuestos antidiabéticos ${ }^{21}$. Los modelos manipulados farmacológicamente incluyen la administración de GTG en ratones, la administración de bajas dosis de aloxán (ALX) y estreptomicina (STZ) en ratas y la administración neonatal de STZ en ratas. La administración de GTG actúa en el centro 
del apetito a nivel del hipotálamo, induciendo entre otras características ya comentadas hiperfagia ${ }^{61}$. Hasta ahora, el ALX y la STZ se consideran los compuestos más potentes para la investigación de la DMT2, empleándose ambos como análogos citotóxicos de la glucosa. El ALX destruye selectivamente las células $\beta$ pancreáticas, aunque hoy en día su uso es menor en comparación con la STZ debido a su menor eficacia, estabilidad, reproducibilidad de resultados y efectos secundarios ${ }^{24,46}$. Por otro lado, la STZ provoca alteraciones en el transporte de la glucosa, en la actividad de la glucocinasa y degradación de múltiples cadenas de $\mathrm{ADN}^{46}$. Existen también modelos mixtos, en los cuales, por ejemplo, se combina la administración de una dieta alta en grasa para inducir resistencia a la insulina seguida de la administración de STZ para provocar una disfunción de las células $\beta$ pancreáticas ${ }^{46}$. Dentro de este grupo se incluye también la administración de nicotinamida seguida de STZ, ya que la primera sirve como agente protector frente al daño pancreático causado por STZ, al igual que evita la depleción del dinucleótido nicotinamida adenina producida por STZ46. Como ventajas de los modelos farmacológicos podrían señalarse que la administración de ALX o STZ provoca una pérdida selectiva de células $\beta$ pancreáticas, dejando intactas las células $\alpha$ y $\delta$ pancreáticas. Además, con la administración de estos compuestos la mortalidad no es elevada y las condiciones de mantenimiento no son excesivamente caras $^{21}$. Como desventajas podría señalarse que en estos animales el desarrollo de hiperglucemia se debe principalmente a una deficiencia de insulina más que a una resistencia a esta. Además, son tratamientos a largo plazo, que emplean sustancias que pueden ser dañinas para otros órganos y en los que es posible observar gran variabilidad de resultados en los estudios realizados ${ }^{21}$.

Los modelos obtenidos mediante manipulación quirúrgica incluyen las ratas pancreatomizadas parciales, en las cuales la diabetes es causada por la pérdida casi total de las células $\beta$ pancreáticas productoras de insulina y cierta capacidad de regeneración posterior ${ }^{22}$. Como ventajas podrían destacarse que con el empleo de esta técnica se evita la toxicidad en otros órganos generada con los métodos farmacológicos y además se asemeja a la DMT2 humana, ya que se consigue una reducción de la masa de células $\beta$ pancreáticas ${ }^{15,46}$. Como desventajas, destacar la complejidad de la técnica, reflejada en la alta mortalidad, la variable capacidad de regeneración del páncreas remanente y en ocasiones, la eliminación de otros tipos de células, importantes en la regulación de la homeostasis de la glucosa ${ }^{20,46}$. Los modelos manipulados genéticamente incluyen el ratón knockout receptor adrenérgico $\beta_{3}$, el ratón knockout para el factor neurotrófico derivado del cerebro (BDNF), el ratón knockout para el receptor de la insulina (IR), el ratón knockout para el sustrato del receptor de insulina (IRS), como IRS-1 e IRS-2, el ratón knockout para el transportador de glucosa 4 (GLUT4) y el ratón transgénico proteína polipéptido amiloide de los islotes pancreáticos humanos (hIAPP). Los receptores adrenérgicos $\beta 3$ son abundantes en el tejido adiposo pardo, y aunque todavía no se conocen exactamente sus mecanismos de actuación, varios estudios han observado que una disminución en su función podría contribuir a la disminución del gasto energético y obesidad. Además, recientemente se ha reportado que una mutación en los receptores $\beta_{3}$ adrenérgicos humanos está asociada con la obesidad, disminución del gasto energético, reducción de la sensibilidad a la insulina y DMT224,62. El BDNF es una proteína conocida por sus acciones en el sistema nervioso central, pudiendo ser además un regulador de la ingesta de alimentos y metabolismo energético, reduciendo la ingesta de alimentos y las concentraciones de glucosa en sangre ${ }^{64}$. Concretamente, un estudio demuestra que cuando ratones knockout BDNF eran mantenidos en un régimen de ayuno intermitente, sus anormalidades en la regulación de la 
glucosa y la obesidad mejoraban. Además, se encontró un aumento en los niveles de BDNF cerebral en comparación con aquellos ratones controles con dieta ad libitum ${ }^{63}$. El receptor de insulina promueve la captación de glucosa en varios tejidos. Dado que la interrupción de la señalización de la insulina en diferentes tejidos contribuye a la patogénesis de la DMT2, se han estudiado varios modelos knockout para el receptor de insulina ${ }^{64}$. Las proteínas del IRS transmiten señales de los receptores de insulina y del factor de crecimiento similar a la insulina 1 (IGF-1) y median los efectos pleiotrópicos de la insulina y el IGF-1, incluida la regulación de la homeostasis de la glucosa y el crecimiento y la supervivencia de las células ${ }^{50}$. El GLUT4 desempeña un papel importante en la captación de glucosa en tejidos sensibles a la insulina. Estudios tanto en humanos como en roedores han demostrado que hay una disminución en el transporte de glucosa en sujetos diabéticos en comparación con sujetos control ${ }^{50,65}$. El polipéptido amiloide de los islotes humanos está implicado en la fisiopatología de la DMT2, al formar en el páncreas unos depósitos conocidos como amiloide del islote, los cuales han sido observados en más del $80 \%$ de pacientes con $\mathrm{DMT}_{2}^{22,66}$. Como ventajas podrían señalarse que permiten el estudio del efecto de un solo gen o de sus respectivas mutaciones en la DMT2, a la vez que facilitan la comprensión de la complejidad genética de la DMT2. Como desventajas, se trata de modelos que requieren grandes inversiones económicas ${ }^{21}$.

\section{CONCLUSIONES}

La utilización de modelos animales desempeña un papel esencial en el estudio de las causas de la diabetes y como herramienta con la que identificar y evaluar diferentes estrategias terapéuticas.

Aunque no existe ningún modelo animal que abarque todas las características de la enfermedad en humanos, los empleados actualmente actúan como herramientas para investigar cambios genéticos, endocrinos, metabólicos, morfológicos y mecanismos subyacentes que ocurren en la DMT2 humana. Por tanto, la selección de un modelo animal en particular (sexo, edad) depende de la elección del investigador, de la disponibilidad de estirpe, del objetivo de la investigación científica, del medicamento que se prueba y de los recursos disponibles.

\section{BIBLIOGRAFÍA}

1. Global report on diabetes [Internet]. Ginebra: World Health Organization; 2016 [consultado: 22-12-2018]. Disponible en: http://www. who.int/diabetes/global-report/en/

2. Kharroubi AT. Diabetes mellitus: the epidemic of the century. World J Diabetes. 2015;6:850-67.

3. Wilcox G. Insulin and insulin resistance. Clin Biochem Rev. 2005;26:19-39.

4. Dimitriadis G, Mitron P, Lambadiari V, Maratou E, Raptis SA. Insulin effects in muscle and adipose tissue. Diabetes Res Clin Pract. 2011; 93:52-9.

5. American Diabetes Association. Diagnosis and classification of diabetes mellitus. Diabetes Care. 2009;32:62-7.

6. Wilmot E, Idris I. Early onset type 2 diabetes: risk factors, clinical impact and management. Ther Adv Chronic Dis. 2014;5:234-44.

7. Papatheodorou K, Papanas N, Banach M, Papazoglou D, Edmonds M. Complications of diabetes. J Diabetes Res. 2016;2016:6989453.

8. Paz-Filho G, Mastronardi C, Wong ML, Licinio J. Leptin therapy, insulin sensitivity, and glucose homeostasis. Indian J Endocrinol Metab. 2012;16:549-55.

9. Thiruvoipati T. Peripheral artery disease in patients with diabetes: epidemiology, mechanisms, and outcomes. World J Diabetes. 2015;6:961-9.

10. Stanhope KL. Sugar consumption, metabolic disease and obesity: the state of the controversy. Crit Rev Clin Lab Sci. 2016;53:52-67.

11. Bringhenti I, Moraes-Teixeira JA, Cunha MR, Ornellas F, Mandarim-de-Lacerda CA, Aguila MB. Maternal obesity during the preconception and early life periods alters pancreatic development in early and adult life in male mouse offspring. PLoS One. 2013;8:e55711.

12. Sami W, Ansari T, Butt NS, Hamid M. Effect of diet on type 2 diabetes mellitus: a review. Int J Health Sci. 2017;11:65-71.

13. Asif $M$. The prevention and control the type-2 diabetes by changing lifestyle and dietary pattern. J Edu Health Promot. 2014;3:1.

14. Via MA, Mechanick JI. Nutrition in type 2 diabetes and the metabolic syndrome. Med Clin North Am. 2016;100:1285-302.

15. Singh MP, Pathak K. Animal models for biological screening of anti-diabetic drugs: an overview. Euro J Exp Bio. 2015;5:37-48.

16. Lukačínová A, Hubková B, Rácz $O$, Ništiar F. Animal models for study of diabetes mellitus. En: Oguntibeju OO, editor. Diabetes mellitus-insights and perspectives. 1. ${ }^{\text {a }}$ edición. Croacia: InTech; 2013. pp. 229-253.

17. Chen D, Wang MW. Development and application of rodent models for type 2 diabetes. Diabetes Obes Metab. 2005;7:307-17.

18. Arias-Díaz J, Balibrea J. Modelos animales de intolerancia a la glucosa y diabetes tipo 2. Nutr Hosp. 2007;22:160-8.

19. Moher D, Shamseer L, Clarke M, Ghersi D, Liberati A, Petticrew M, et al. Preferred reporting items for systematic review and meta-analysis protocols (PRISMA-P) 2015 statement. Syst Rev. 2015;4:1. 
20. Rees DA, Alcolado JC. Animal models of diabetes mellitus. Diabet Med. 2005;22:359-70.

21. Sah SP, Singh B, Choudhary S, Kumar A. Animal models of insulin resistance: a review. Pharmacol Rep. 2016;68:1165-77.

22. King AJ. The use of animal models in diabetes research. Br J Pharmacol. 2012;166:877-94.

23. Wang B, Chandrasekera P, Pippin J. Leptin-and leptin receptor deficient rodent models: Relevance for human type 2 diabetes. Curr Diabetes Rev. 2014;10:131-45.

24. Da Silva Xavier G, Hodson DJ. Mouse models of peripheral metabolic disease. Best Pract Res Clin Endocrinol Metab. 2018;32:299-315.

25. Akash M, Rehman K, Chen S. An overview of valuable scientific models for diabetes mellitus. Curr Diabetes Rev. 2013;9:286-93.

26. Brown L, Panchal SK. Rodent models for metabolic syndrome research. J Biomed Biotechnol. 2011;2011:351982.

27. Sima AAF, Shafrir E. Animal models of diabetes: A primer. Amsterdam, Países Bajos: Harwood Academic Publishers; 2000.

28. Lindström P. The physiology of obese-hyperglycemic mice [ob/ob mice]. ScientificWorldJournal. 2007;7:666-85.

29. Lindström P. $\beta$-Cell function in obese-hyperglycemic mice [ob/ob mice]. Adv Exp Med Biol. 2010;654:463-77.

30. Oana F, Takeda H, Hayakawa K, Matsuzawa A, Akahane S, Isaji M, et al. Physiological difference between obese ( $\mathrm{fa} / \mathrm{fa}$ ) Zucker rats and lean Zucker rats concerning adiponectin. Metabolism. 2005;54:995-1001.

31. Al-Awar A, Kupai K, Veszelka M, Szucs G, Attieh Z, Murlasits Z, et al. Experimental diabetes mellitus in different animal models. J Diabetes Res. 2016;2016:9051426.

32. Ikeda H. KK mouse. Diabetes Res Clin Pract. 1994;24:313-16.

33. Koza RA, Flurkey K, Graunke DM, Braun C, Pan HJ, Reifsnyder PC, et al. Contributions of dysregulated energy metabolism to type 2 diabetes development in NZO/H1Lt mice with polygenic obesity. Metabolism. 2004;53:799-808.

34. Leiter EH, Strobel M, O’Neill A, Schultz D, Schile A, Reifsnyder PC. Comparison of two new mouse models of polygenic type 2 diabetes at the Jackson Laboratory, NONcNZO10Lt/J and TALLYHO/JngJ. J Diabetes Res. 2013;2013:165327.

35. Suzuki W, lizuka S, Tabuchi M, Funo S, Yanagisawa T, Kimura M, et al. A new mouse model of spontaneous diabetes derived from ddY strain. Exp Anim. 1999;48:181-9.

36. Allan MF, Eisen EJ, Pomp D. The M16 mouse: an outbred animal model of early onset polygenic obesity and diabesity. Obes Res. 2004;12: $1397-407$.

37. Kim JH, Stewart TP, Soltani-Bejnood M, Wang L, Fortuna JM, Mostafa OA, et al. Phenotypic characterization of polygenic type 2 diabetes in TALLYHO/JngJ mice. J Endocrinol. 2006;191:437-46.

38. Moran TH. Unraveling the obesity of OLETF rats. Physiol Behav. 2008;94:71-8.

39. Moran TH, Bi S. Hyperphagia and obesity in OLETF rats lacking CCK-1 receptors. Philos Trans R Soc B Biol Sci. 2006;361:1211-8.

40. Akash M, Rehman K, Chen S. Goto-kakizaki rats: its suitability as nonobese diabetic animal model for spontaneous type 2 diabetes mellitus. Curr Diabetes Rev. 2013;9:387-96.

41. Shinohara M, Masuyama T, Shoda T, Takahashi T, Katsuda Y, Komeda $\mathrm{K}$, et al. A new spontaneously diabetic non-obese Torii rat strain with severe ocular complications. Int J Exp Diabetes Res. 2000;1:89-100.

42. Gheibi S, Kashfi K, Ghasemi A. A practical guide for induction of type2 diabetes in rat: incorporating a high-fat diet and streptozotocin. Biomed Pharmacother. 2017;95:605-13.

43. Surwit RS, Feinglos MN, Rodin J, Sutherland A, Petro AE, Opara AE, et al. Differential effects of fat and sucrose on the development of obesity and diabetes in C57BL/6J and A/J mice. Metabolism. 1995;44: 645-51.

44. Heydemann A. An overview of murine high fat diet as a model for type 2 diabetes mellitus. J Diabetes Res. 2016;2016:2902351.
45. Reuter TY. Diet-induced models for obesity and type 2 diabetes. Drug Discov Today Dis Models. 2007;4:3-8.

46. Islam MS, Wilson RD. Experimentally induced rodent models of type 2 diabetes. Methods Mol Biol. 2012;933:161-74.

47. Shafrir E, Ziv E, Kalman R. Nutritionally induced diabetes in desert rodents as models of type 2 diabetes: Acomys cahirinus (spiny mice) and Psammomys obesus (desert gerbil). ILAR J. 2006;47:212-24.

48. Kaiser N, Cerasi E, Leibowitz G. Diet-induced diabetes in the sand rat (Psammomys obesus). Methods Mol Biol. 2012;933:89-102.

49. Matsuo T, Shino A. Induction of diabetic alterations by goldthioglucose-obesity in KK, ICR and C57BL Mice. Diabetologia. 1972;8:391-7.

50. Sone H, Suzuki H, Takahashi A, Yamada N. Disease model: hyperinsulinemia and insulin resistance: part A-targeted disruption of insulin signaling or glucose transport. Trends Mol Med. 2001;7:320-2.

51. King A, Bowe J. Animal models for diabetes: understanding the pathogenesis and finding new treatments. Biochem Pharmacol. 2016; 99:1-10.

52. Christopher RJ, Takeuchi K, Lee B. Rodent models of diabetes. En: Poretsky L, editor. Principles of diabetes mellitus. 3. a edidión. EE.UU., Boston: Springer; 2017. pp. 165-181.

53. Liu Y, Meyer C, Xu C, Weng H, Hellerbrand C, Dooley S, et al. Animal models of chronic liver diseases. Am J Physiol Gastrointest Liver Physiol. 2013;304:449-68.

54. Vogel HG, Vogel WH. Genetically obese animals. En: Vogel HG, editor. Drug discovery and evaluation: pharmacological assays. EE.UU., Boston: Springer; 2008. pp. 1614-1622.

55. Joost HG. Personalized prevention of type 2 diabetes. En: Kok F, Bouwman L, Desiere F, editores. Personalized nutrition: principles and applications. EE.UU., Boca Ratón: CRC Press; 2008. pp. 61-74.

56. Okuma C, Ishii Y, Ohta T. Current status of medical therapy and new targets for anti obesity drug development. En: Atta-ur-Rahman, Choudhary MI, editores. Anti obesity drug discovery and development. 1. ${ }^{a}$ edición. UAE, Sharjah: Bentham Science; 2017. pp. 3-64.

57. Clee SM, Attie AD. The genetic landscape of type 2 diabetes in mice. Endocr Rev. 2008;28:48-83.

58. Najafian B, Alpers CE. Pathology of the kidney in diabetes. En: Roelofs JJ, Vogt L, editores. Diabetic nephropathy. Pathophysiology and clinical aspects. 1. ${ }^{\text {a }}$ edición. EE.UU., Boston: Springer; 2018. pp. 113-143.

59. Weksler-Zangen S, Yagil C, Zangen DH, Ornoy A, Jacob HJ, Yagil, Y, et al. The newly inbred cohen diabetic rat: a nonobese normolipidemic genetic model of diet-induced type 2 diabetes expressing sex differences. Diabetes. 2001;50:2521-9.

60. Brito-Casillas Y, Melián C, Wägner AM. Study of the pathogenesis and treatment of diabetes mellitus through animal models. Endocrinol Nutr. 2016;63:345-53.

61. Karasawa H, Takaishi K, Kumagae Y. Obesity induced diabetes in mouse strains treated with gold thioglucose: a novel animal model for studying $\beta$ cell dysfunction. Obesity. 2011;19:514-21.

62. Lowell BB, Flier JS. Brown adiopose tissue, $\beta 3$ adrenergic receptors, and obesity. Annu Rev Med. 1997;48:307-16.

63. Duan W, Guo Z, Jiang H, Ware M, Mattson MP. Reversal of behavioral and metabolic abnormalities, and insulin resistance syndrome, by dietary restriction in mice deficient in brain-derived neurotrophic factor. Endocrinology. 2003;144:2446-53.

64. Kitamura T, Kahn CR, Accili D. Insulin receptor knockout mice. Annu Rev Physiol. 2003;65:313-32.

65. Stenbit AE, Tsao TS, Li J, Burcelin R, Geenen DL, Factor SM, et al. GLUT4 heterozygous knockout mice develop muscle insulin resistance and diabetes. Nat Med. 1997;3:1096-101.

66. Höppener JW, Jacobs HM, Wierup N, Sotthewes G, Sprong M, de Vos $P$, et al. Human islet amyloid polypeptide transgenic mice: in vivo and ex vivo models for the role of hIAPP in type 2 diabetes mellitus. Exp Diabetes Res. 2008;2008:697035. 